\title{
Dirofilariose sub-cutanea dos cães no Brasil *
}

\author{
por \\ Herman Lent e J. F. Teixeira de Freitas
}

(Com 4 figuras no texto)

O estudo de um filarideo encontrado no Estado de São Paulo (Instituto Butantan) no lecido sub-culaneo de um cão, em 1918, incluido agora na collecção helminthologica do Instituto Oswaldo Cruz sob o numero 9576, nos proporcionou a conslatação, pela primeira vez no Brasil, de mais um helmintho capaz de parasitar os cães.

O interesse deste encontro torna-se ainda maior porquanto o helmintho em questão, do qual não existe estudo perfeito, vinha sendo confundido com outros do mesmo genero, descriptos de animaes de grupos zoologicos diversos, taes como Tayassus tajacu (L.), Erethizon dorsalus L., Hyaena (Crocotta) crocuta (Erxl.) e Lynx canadensis.

Sob o nome de Dirofilaria repens Railliet \& Henry, 1911, estes autores tornaram conhecida uma especie estudada preliminarmente por Fuelleborn, em 1908, e observada por elles de material recolhido no tecido subcutaneo de um cão pelo professor Bonvicini, em Bolonha.

No mesmo anno Bauche \& Bernard publicaram os casos clinicos de dois cães do Annam (Indochina franceza), attingidos por essa filariose e que possuiam microfilarias extremamente numerosas no sangue e adultos, machos e femeas, localisados exclusivamente no tecido cellular sub-cutaneo, com manifestações de prurido sem lesões cutaneas superficiaes, cachexia, grande emmagrecimento e anemia, e asthenia profunda. No primeiro cão, que após aulopsia não revelou filarideos em outra qualquer região do corpo, a não ser no tecido cellular sub-cutaneo, principalmente no tecido mais f́rouxo da face interna da raiz dos membros, onde a pelle é mais fina e quasi completamente desprovida de pellos, encontraram 8 machos e 14 femeas gravidas. No segundo cão, tambem dos annamitas, foram observados 9 machos e 16 femeas e nenhum outro filarideo em outro local do corpo. Estes autores, que descreveram o parasito sem o denominar, o approximaram, entretanto, de

* Recebido para publicação a 6 de Julho de 1937 e dado á publicidade em Setembro de 1937. 
Dirofilaria immilis (Leidy, 1856), tambem causador de helminthose em cães, mas de localisação preferida no coração ou nos vasos.

Railliet \& Henry, ainda em 1911, tiveram a opportunidade de examinar o material que serviu de base ao estudo de Bauche \& Bernard, que reclificam em alguns pontos. Summariam as observações até então registradas de filarideos sub-culaneos, taes como as de Frcolani (1875); Rivolla (1878); Vachelta (citado por Rivolta); Grassi (1888); Ninni (1889), caso que Stossich dá como causado pela Dirofilaria acutiuscula (Molin, 1858); Galli-Valerio (1897); Feltick (1899); Fuelleborn (1908); Bonvicini (1910); e Gogel (1910); só puderam, entretanto, constatar a identidade do parasilo, nas observações de Fuelleborn, que o descreveu, e na de Bonvicini que enviou o malerial aos autores irancezes.

Mais tarde, em 1913, Bernard \& Bauche provam a transmissão de Dirofilaria repens pelo Stegomyia aegypı́i e chamam a attenção para a grande porcentagem de cães infestados, em relação ao parasitismo por D. immitis, no Annam, e a presença de fócos nas cidades, justamente em connexão com o modo de vida do mosquilo transmissor.

Apezar de todas as differenças assignaladas atravez dos trabalhos que acinia synthelisamos, o helmintho não ficou bem conhecido e, por conseguinte, não acceilo pela generalidade dos helminthologistas, devendo ter sido algumas vezes registrado como D. immitis. Sómente em 1927 Vogel publicou um estudo comparativo das duas especies proximas D. immitis e D. repens - e figuras da extremidade caudal dos machos.

Em 1930, Skrjabin, Althausen \& Schulman publicam um trabalho, que infelizmente não possuimos, descrevendo um caso no qual a Dirofilaria repens foi extrahida de um nodulo sub-cutaneo localisado na palpebra inferior direila de uma mulher, na Russia. Deve tratar-se de um caso de parasilismo accidental, a determinação do helmintho tendo sido feita pelo conhecido helminthologista russo.

Tralados modernos de helminthologia, taes como os de Mönnig (1934) e Neveu-Lemaire (1936), teem referido este filarideo como Dirofilaria acutiuscula (Molin, 1858) transcrevendo a descripção de Railliet \& Henry para $D$. repens, o que não representa a realidade, conforme de-monstramos em trabalho publicado este anno. Os dois helminthos são facilmente distinguidos, este erro tendo se repetido por causa da identificação feita por Stossich (1890) do parasito que Ninni encontrou sob a pelle de um cão.

Tambem Chitwood, aliás o primeiro a denominar Filaria acutiuscula Molin, 1858 como Dirofilaria, o fez baseado em material proveniente de Lynx canadensis, asseverando ser aquelle parasilo, original de porcos do malto sul-americanos [Tayassus tajacu (L.) e T. albirostris (Illig.)], 
o mesmo helmintho que Railliet \& Henry descreveram do cão sob o nome de $D$. repens.

Com o material que nesta publicação descrevemos, addicionado ao estudo que já publicamos sobre Dirofilaria acutiuscula (Molin, 1858), estamos em condicções de poder asseverar que não se trata, absolutamente, de uma só especie; acreditamos que os exemplares que Chitwood observou em Lynx canadensis possam pertencer a uma terceira especie.

Dirofilaria repens Railliet \& Henry, 1911.

(Figs. 1-4).

Comprimento:- Femeas 140 a $150 \mathrm{~mm}$.

Largura:-Femeas 0,447 a $0,552 \mathrm{~mm}$.

Corpo com cuticula branca, nitidamente estriada longitudinalmente. Es-

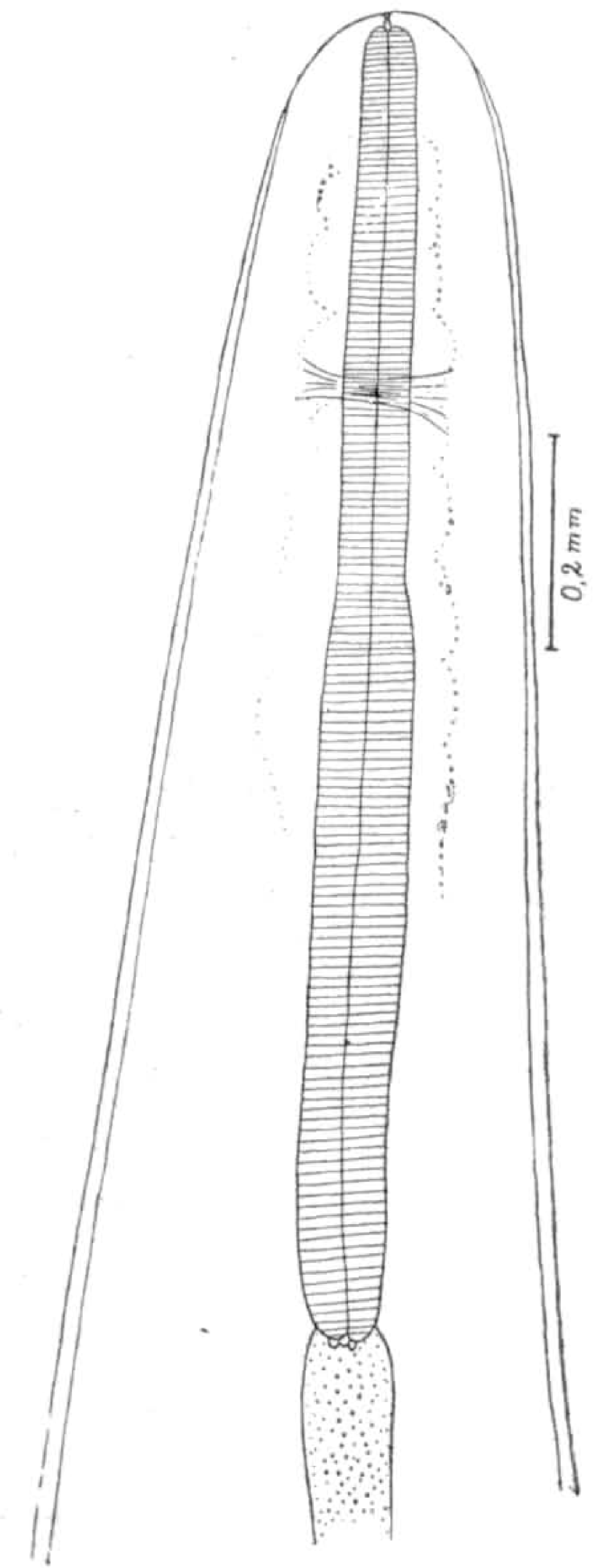

Fig. 1 - Dirofilaria repens Railliet \& Henry, 1911. Extremidade anterior do macho. Original.

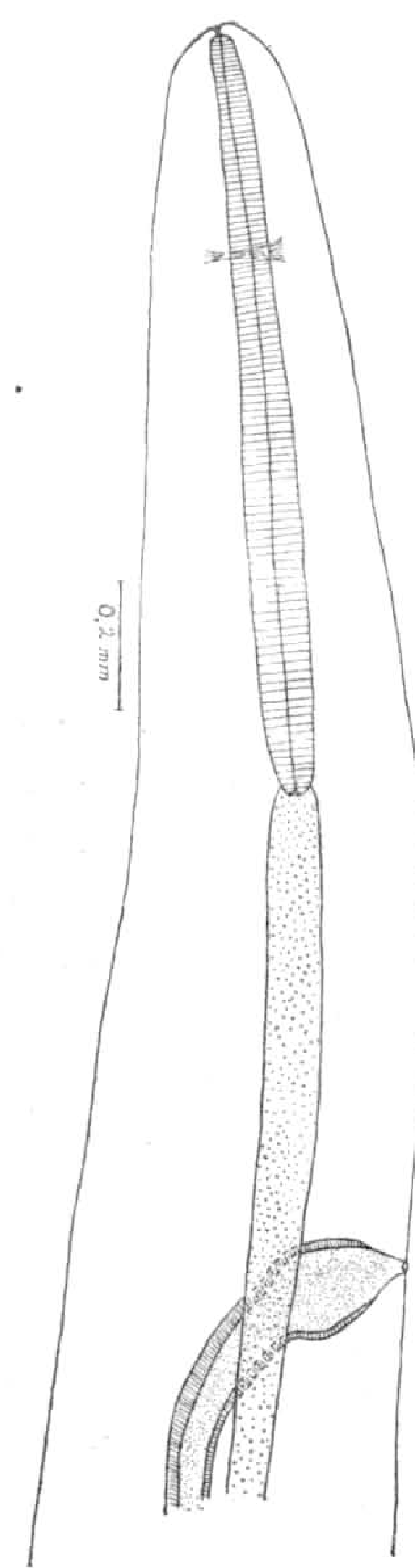

Fig. 2 - Dirofilaria repens Railliet \& Henry, 1911. Extremidade anterior da femea. Original. 
trias transversaes delicadas e nitidas. Extremidades afiladas. Extremidade cephalica desprovida de papillas, apresentando uma cuticula um pouco espessada, sendo a superficie de aspecto granuloso. Esse espessamento da cuticula se extende até o nivel de um reforço interno, que parte das paredes do corpo e circumda o esophago. Bocca simples; capsula buccal vestigial. Esophago dividido em duas porções, medindo nas femeas 1,05 a $1,53 \mathrm{~mm}$. de comprimento, sendo 0,49 a $0,54 \mathrm{~mm}$. para a porção anterior. O esophago é separado do intestino por 3 pequenas valvulas. Annel nervoso situado a 0,304 a $0,368 \mathrm{~mm}$. da extremidade anterior nas femeas. Papillas cervicaes e póro excretor não evidenciados.

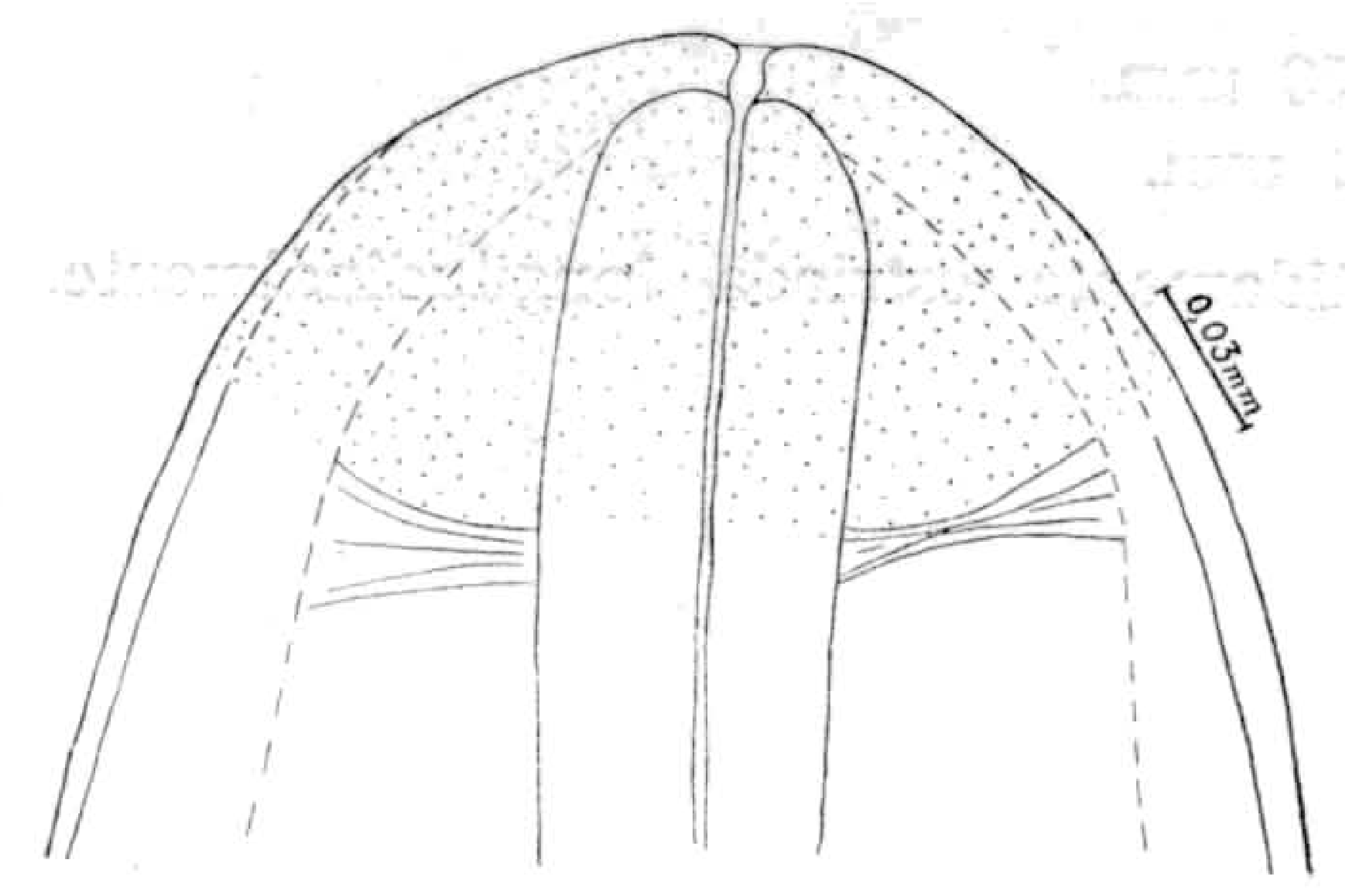

Fig. 3 - Dirofilaria repens Railliet \& Henry, 1911. Detalhe da extremidade cephalica. Original.

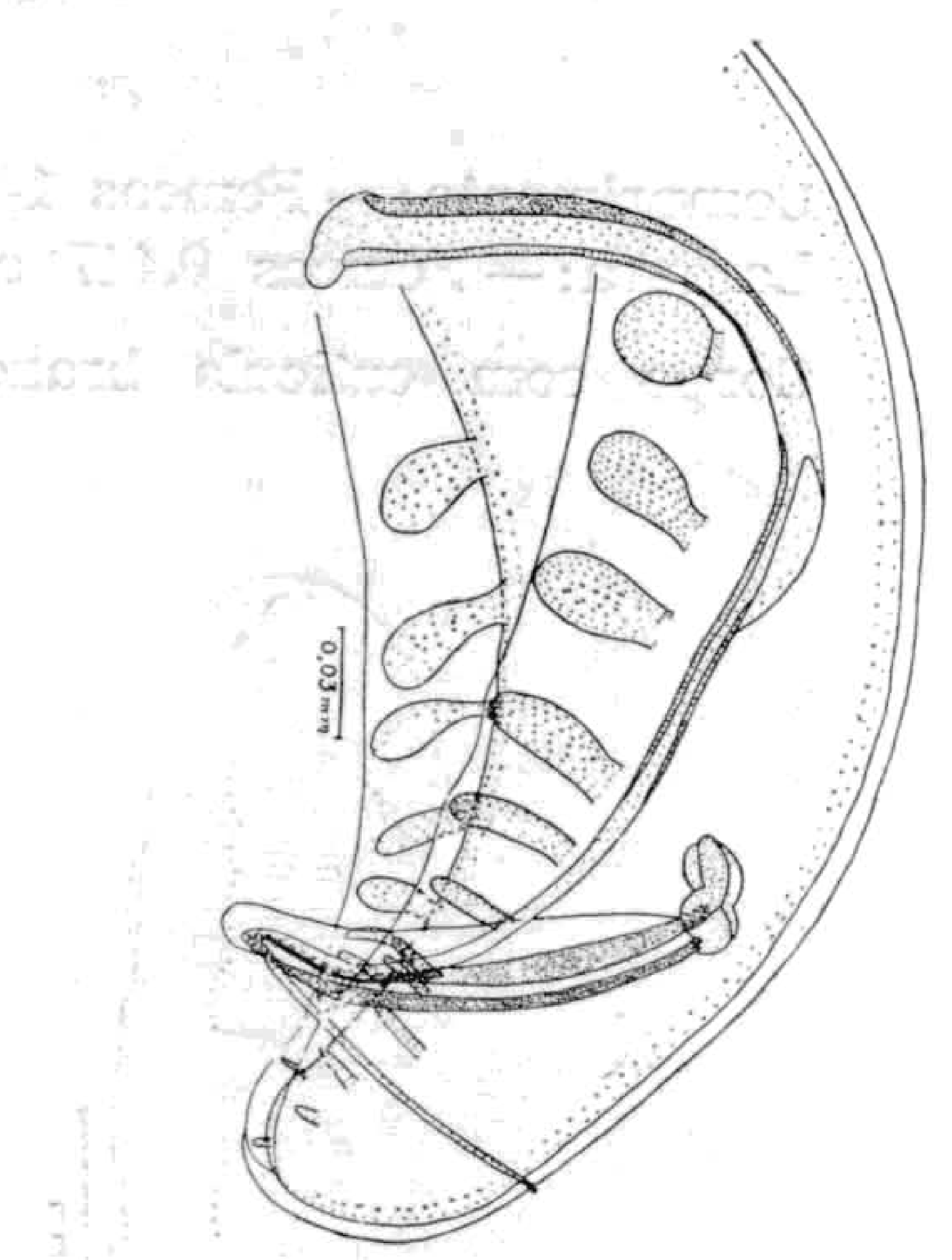

Fig. 4-Dirofilaria repens Railliet \& Henry, 1911. Extremidade caudal do macho. Orig.

Femeas didelphas, opisthodelphas, viviparas, com vulva ás vezes de labios levemente salientes, situada a 1,84 a $1,92 \mathrm{~mm}$. da extremidade anterior. Vagina longa, com cerca de $3,42 \mathrm{~mm}$. de comprimento. Em alguns exemplares a vagina e o ovejector formam numerosas alças que se extendem para diante, voltando-se depois para traz, ligando-se então aos uteros, que occupam quasi toda a cavidade do corpo, formando ou não alças. Ovarios ennovelados, situados na extremidade posterior do corpo. Intestino fino, mais ou menos rectilineo. Anus praticamente terminal. Cauda de apice obtuso, levemente curvada ventralmente.

Macho com espiculos desiguaes e não semelhantes. Gubernaculo ausente. O espiculo maior mede $0,430 \mathrm{~mm}$. de comprimento, apresentando a base levemente dilatada e a ponta muito afilada. O espiculo menor é mais chitinisado, mede $0,175 \mathrm{~mm}$. de comprimento, apresenta a base bastante dilatada e a ponta obtusa, possuindo, ainda, uma larga aza membranosa lisa que o percorre lateraìmente da base á ponta, envolvendo-a. A relação entre os espiculos é approximadamente de $1: 2,5$. Cauda levemente curvada ventralmente, com azas lateraes e papillas pedunculadas oblongas, situadas da seguinte maneira: 6 pre- 
anaes, 2 ad-anaes e 3 post-anaes de um lado; no outro lado as ad- e postanaes são identicas, porém, as pre-anaes são sómente 5 . Orificio cloacal situado a $0,072 \mathrm{~mm}$. do apice caudal, que é obtuso. O unico fragmento de macho que examinamos media $27,75 \mathrm{~mm}$. de comprimento.

Habitat : - Tecido sub-cutaneo de Canis familiaris L.

Proveniencia : - S. Paulo (Estado de São Paulo), Brasil.

Examinamos 5 femeas inteiras e um macho fragmentado.

Como é de todo interesse pratico a pesquisa dos parasitos de animaes domesticos no Brasil, dos quaes a lista de Pinto \& Almeida (1935) dará bôa orientação, aconselhamos a differenciação das duas especies de Dirofilaria encontradas nos cães, pelo quadro pratico differencial abaixo. Note-se que já pelo exame do sangue peripherico e observação das microfilarias é possivel diagnosticar-se, além da verificação dos symptomas apresentados, a entidade morbida que o animal possa apresentar.

\section{Quadro 1}

\begin{tabular}{|c|c|c|}
\hline & $\begin{array}{l}\text { Dirofilaria immitis } \\
\text { (Leidy, 1856) }\end{array}$ & $\begin{array}{c}\text { Dirofilaria repens } \\
\text { Railliet \& Henry, } 1911\end{array}$ \\
\hline Comprimento do corpo $\left\{\begin{array}{l}q \\
0^{x}\end{array}\right.$ & $\begin{array}{l}150-300 \mathrm{~mm} . \\
120-180 \mathrm{~mm} .\end{array}$ & $\begin{array}{l}100-170 \mathrm{~mm} . \\
48-70 \mathrm{~mm} .\end{array}$ \\
\hline Largura do corpo $\left\{\begin{array}{l}9 \\
\sigma^{\infty}\end{array}\right.$ & $\begin{array}{l}1-1,3 \mathrm{~mm} \\
0,6-0,9 \mathrm{~mm}\end{array}$ & $\begin{array}{l}0,45-0,65 \mathrm{~mm} . \\
0,37-0,45 \mathrm{~mm} .\end{array}$ \\
\hline Extremidade cephalica & com papillas & sem papillas \\
\hline Espiculos $\quad\left\{\begin{array}{l}\text { grande } \\
\text { pequeno }\end{array}\right.$ & $\begin{array}{l}0,30-0,355 \mathrm{~mm} . \\
0,175-0,226 \mathrm{~mm} .\end{array}$ & $\begin{array}{l}0,43-0,59 \mathrm{~mm} . \\
0,175-0,21 \mathrm{~mm} .\end{array}$ \\
\hline Relação entre os espiculos & $1,6: 1$ & $2,5: 1$ \\
\hline $\begin{array}{l}\text { Distancia da vulva á } \\
\quad \text { extremidade anterior }\end{array}$ & $2,35-3,40 \mathrm{~mm}$. & $1,5-1,9 \mathrm{~mm}$ \\
\hline Situação do anus & $\begin{array}{l}\text { a alguma distancia do apice } \\
\text { caudal }\end{array}$ & praticamente terminal \\
\hline Embryð̃es & $\begin{array}{l}0,180-0,285 \mathrm{~mm} \\
\underset{0,005 \mathrm{~mm}}{X}\end{array}$ & 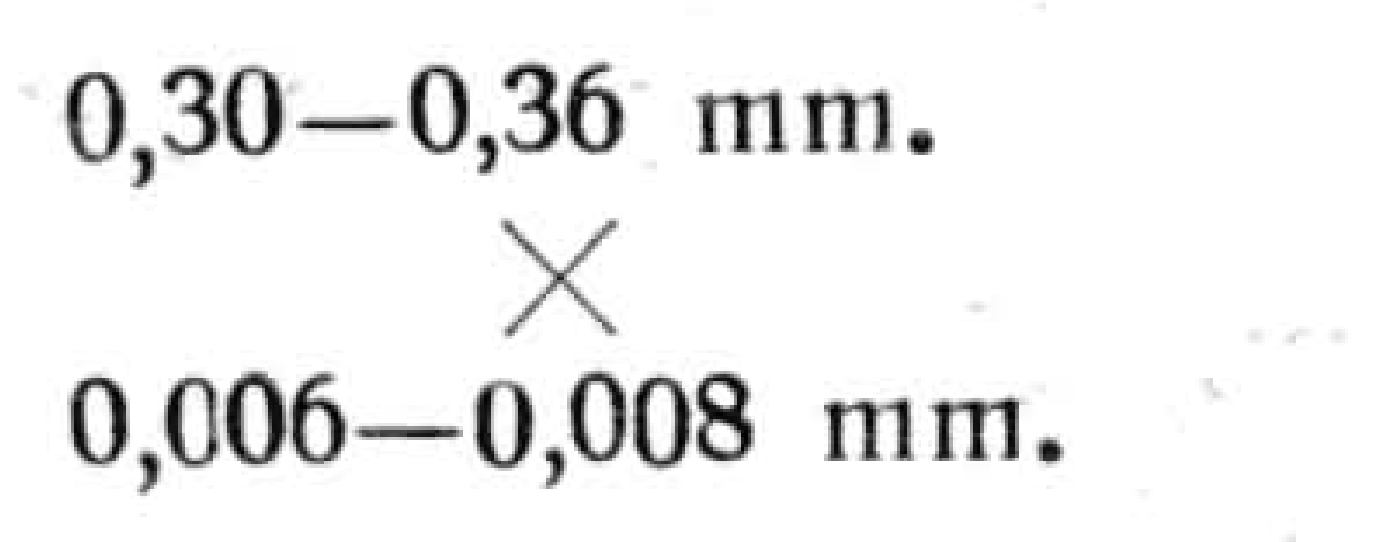 \\
\hline Localisação frequente & vascular & sub-cutanea \\
\hline
\end{tabular}




\section{BIBLIOGRAPHIA}

Bauche, J. \& Bernard, P. N.

1911. Sur deux cas de filariose du chien. Bull. Soc. Path. Exot., 4 (7) : 478482.

Bernard, P. N. \& Bauche, J.

1913. Conditions de propagation de la filariose sous-cutanée du chien. Stegomyia fasciata hôte intermediaire de Dirofilaria repens. Bull. Soc. Path. Exot., 6 (1) : 89-99, figs. 1-9.

Chitwood, B. G.

1933. Note on a genus and species of Nematode form Lynx canadensis. Jour. Parasitol., 20 (1) : 63.

Lent, H. \& Freitas, J. F. Teixeira de

1937. Contribuição ao estudo do genero Dirofilaria Railliet \& Henry, 1911. Mem. Inst. Oswaldo Cruz, 32 (1) : 37-54, 7 ests., 22 figs.

MönNig, H. O.

1934. Veterinary Helminthology and Entomology. XVI. 402 pp., 264 figs. Londres.

Neveu-Lemaire, M.

1936. Traité d'Helminthologie Médicale et Vétérinaire. XXIII. 1514 pp., 787 figs. Paris.

Pinto, C. \& Almeida, J. Lins de

1935. Sinopse dos helmintos dos animais domesticos do Brasil. O Campo, 6 (8) : 54-63, 10 figs.

Railliet, A. \& Henry, A.

1911. Sur une filaire péritoneale des porcins. Bull. Soc. Path. Exot., 4 (6) : 386-389.

1911. Remarques au suject des deux notes de MM. Bauche \& Bernard. Bull. Soc. Path. Exot., 4 (7) : 485-488.

Skruabin, K. I., Althausen, A. J. \& Schulmann, E. S.

1930. First case of Dirofilaria repens from man. Trop. Med, \& Vet., Moscow, 8 (2) : 9-11, 4 figs. Ref. in Trop. Dis. Bull., 1931, 28 : 683.

Stossich, M.

1890. Elminti veneti raccolti dal Dr. Alessandro Conte De Ninni. Boll. Soc. Adriat. di Sci. Nat. in Trieste, 12 : 49-56.

VOGEL, H.

1927. Beitraege zur Anatomic der Gattungen Dirofilaria und Loa. Centralbl. f. Bakt., Orig., 102 (1-3) : 81-89, 13 figs. 